TRANSACTIONS OF THE

AMERICAN MATHEMATICAL SOCIETY

Volume 355 , Number 7 , Pages 2973-2989

S 0002-9947(03)03257-4

Article electronically published on March 14, 2003

\title{
STRONGLY INDEFINITE FUNCTIONALS AND MULTIPLE SOLUTIONS OF ELLIPTIC SYSTEMS
}

\author{
D. G. DE FIGUEIREDO AND Y. H. DING
}

Abstract. We study existence and multiplicity of solutions of the elliptic system

$$
\begin{cases}-\Delta u=H_{u}(x, u, v) & \text { in } \Omega, \\ -\Delta v=-H_{v}(x, u, v) & \text { in } \Omega, \quad u(x)=v(x)=0 \quad \text { on } \partial \Omega,\end{cases}
$$

where $\Omega \subset \mathbb{R}^{N}, N \geq 3$, is a smooth bounded domain and $H \in \mathcal{C}^{1}\left(\bar{\Omega} \times \mathbb{R}^{2}, \mathbb{R}\right)$. We assume that the nonlinear term

$$
H(x, u, v) \sim|u|^{p}+|v|^{q}+R(x, u, v) \text { with } \lim _{|(u, v)| \rightarrow \infty} \frac{R(x, u, v)}{|u|^{p}+|v|^{q}}=0,
$$

where $p \in\left(1,2^{*}\right), 2^{*}:=2 N /(N-2)$, and $q \in(1, \infty)$. So some supercritical systems are included. Nontrivial solutions are obtained. When $H(x, u, v)$ is even in $(u, v)$, we show that the system possesses a sequence of solutions associated with a sequence of positive energies (resp. negative energies) going toward infinity (resp. zero) if $p>2$ (resp. $p<2$ ). All results are proved using variational methods. Some new critical point theorems for strongly indefinite functionals are proved.

\section{INTRODUCTION AND MAIN RESULTS}

Consider the following elliptic system:

$$
\left\{\begin{aligned}
-\Delta u & =H_{u}(x, u, v) & & \text { in } \Omega, \\
-\Delta v & =-H_{v}(x, u, v) & & \text { in } \Omega, \\
u(x) & =v(x)=0 & & \text { on } \partial \Omega,
\end{aligned}\right.
$$

where $\Omega \subset \mathbb{R}^{N}, N \geq 3$, is a smooth bounded domain and $H: \bar{\Omega} \times \mathbb{R}^{2} \rightarrow \mathbb{R}$ is a $\mathcal{C}^{1}$ function. Here $H_{u}$ denotes the partial derivative of $H$ with respect to the variable $u$. Writing $z:=(u, v)$, we suppose $H(x, 0) \equiv 0$ and $H_{z}(x, 0) \equiv 0$. Then $z=0$ is a trivial solution of the system. In this paper we discuss the existence of nontrivial solutions. Roughly speaking, we are mainly interested in the class of Hamiltonians $H$ such that

$$
H(x, u, v) \sim|u|^{p}+|v|^{q}+R(x, u, v) \text { with } \lim _{|z| \rightarrow \infty} \frac{R(x, u, v)}{|u|^{p}+|v|^{q}}=0,
$$

where $1<p<2^{*}:=2 N /(N-2)$ and $q>1$. The most interesting results obtained here refer to the case when $q \geq 2^{*}$, which correspond to critical and supercritical problems. The case when $q<2^{*}$ has been studied by Costa and Magalhães [5],

Received by the editors June 18, 2001.

2000 Mathematics Subject Classification. Primary 35J50; Secondary 58E99.

Key words and phrases. Elliptic system, multiple solutions, critical point theory. 
6] and Benci and Rabinowitz [3]. See also Bartsch and De Figueiredo [2, De Figueiredo and Magalhães [7, De Figueiredo and Felmer [8] and Hulshof and van der Vorst [11], where similar systems also leading to strongly indefinite functionals have been studied. However, only subcritical systems have been considered in those papers.

Letting $2_{*}=2^{*} /\left(2^{*}-1\right)=2 N /(N+2)$, we assume that $H(x, z)$ satisfies the following condition:

$\left(H_{0}\right)$ there are $p \in\left(1,2^{*}\right), q \in(1, \infty)$ and $\tau \in\left(1,1+q / 2_{*}\right)$ such that, for all $(x, z)$,

$$
\left|H_{u}(x, u, v)\right| \leq \gamma_{0}\left(1+|u|^{p-1}+|v|^{\tau-1}\right)
$$

and

$$
\left|H_{v}(x, u, v)\right| \leq \gamma_{0}\left(1+|u|^{p-1}+|v|^{q-1}\right) .
$$

In all hypotheses on $H(x, z)$ the $\gamma_{i}$ 's denote positive constants independent of $(x, z)$. We note that if $q<2^{*}$, then $2_{*}<q /(q-1)$, i.e., $q-1<q / 2_{*}$. Hence, it is possible that $q \leq \tau<1+q / 2_{*}$. However, if $q \geq 2^{*}$, then $\tau<q$. Furthermore, we remark that $\tau$ can be very large, if $q$ is sufficiently large.

In addition, we need distinct conditions on $H$ corresponding to the cases when $p>2, p<2$ or $p=2$.

First, consider the case when $p>2$. In this case, we assume the following three conditions:

$\left(H_{1}\right)$ there are $\mu>2, \nu>1$ and $R_{1} \geq 0$ such that

$$
\frac{1}{\mu} H_{u}(x, z) u+\frac{1}{\nu} H_{v}(x, z) v \geq H(x, z) \text { whenever }|z| \geq R_{1},
$$

with the provision that $\nu=\mu$ if $q>2$;

$\left(H_{2}\right)$ there are $2_{*}(p-1) \leq \alpha \leq p$ and $2_{*}(\tau-1)<\beta$ such that

$$
H(x, z) \geq \gamma_{1}\left(|u|^{\alpha}+|v|^{\beta}\right)-\gamma_{2} \text { for all }(x, z),
$$

and $\beta=q$ if $q>2^{*}$;

$\left(H_{3}\right) H(x, 0, v) \geq 0$ and $H_{u}(x, u, 0)=o(|u|)$ as $u \rightarrow 0$ uniformly in $x$.

We prove the following results.

Theorem 1.1. Let $\left(H_{0}\right)$ be satisfied with $p>2$. If $\left(H_{1}\right)-\left(H_{3}\right)$ hold, then (E) has at least one nontrivial solution.

In order to provide some more transparent hypotheses under which the above result holds, we next present some conditions on $H$ that are sufficient for $\left(H_{0}\right)$, $\left(H_{1}\right)$ and $\left(H_{2}\right)$ to hold:

$\left(H_{0}^{\prime}\right)$ there are $p \in\left(1,2^{*}\right)$ and $q \in(2, \infty)$ such that, for all $(x, z)$,

$$
\left|H_{u}(x, u, v)\right| \leq \gamma_{0}\left(1+|u|^{p-1}+|v|^{\frac{q}{2}-1}\right)
$$

and

$$
\left|H_{v}(x, u, v)\right| \leq \gamma_{0}\left(1+|u|^{p-1}+|v|^{q-1}\right) ;
$$

$\left(H_{1}^{\prime}\right)$ there are $\mu>2$ and $R_{1} \geq 0$ such that

$$
H_{u}(x, z) u+H_{v}(x, z) v \geq \mu H(x, z) \text { whenever }|z| \geq R_{1} ;
$$

$\left(H_{2}^{\prime}\right)$ for $p$ and $q$ as above,

$$
H(x, z) \geq \gamma_{1}\left(|u|^{p}+|v|^{q}\right)-\gamma_{2} \quad \text { for all }(x, z) .
$$


Theorem 1.1'. Let $\left(H_{0}^{\prime}\right)$ be satisfied with $p>2$. If $\left(H_{1}^{\prime}\right),\left(H_{2}^{\prime}\right)$, and $\left(H_{3}\right)$ hold, then (E) has at least one nontrivial solution.

Theorem 1.2. Let $\left(H_{0}\right)$ be satisfied with $p>2$. If $H(x, z)$ is even in $z$ and satisfies $\left(H_{1}\right)$ and $\left(H_{2}\right)$, then $(\mathrm{E})$ has a sequence $\left(z_{n}\right)$ of solutions with energies $I\left(z_{n}\right):=\int_{\Omega}\left(\frac{1}{2}\left(\left|\nabla u_{n}\right|^{2}-\left|\nabla v_{n}\right|^{2}\right)-H\left(x, z_{n}\right)\right)$, going to $\infty$ as $n \rightarrow \infty$.

In order to describe the other results, let $\sigma(-\Delta)$ denote the set of all eigenvalues of $\left(-\Delta, H_{0}^{1}(\Omega)\right): \lambda_{1}<\lambda_{2} \leq \lambda_{3} \leq \cdots$.

We now consider the case when $p<2$. We make the following assumptions:

$\left(H_{4}\right)$ there are $\mu \in(1,2), \quad \nu \geq 2$ and $\gamma_{3} \geq 0 \quad\left(\gamma_{3}=0\right.$, if $\left.q>2^{*}\right)$ such that

$$
H(x, u, v) \geq \frac{1}{\mu} H_{u}(x, u, v) u+\frac{1}{\nu} H_{v}(x, u, v) v-\gamma_{3} \quad \text { for all }(x, z) ;
$$

$\left(H_{5}\right)$ there are $\alpha \in(1,2)$ and $\delta \in(0,1 / 2)$ such that $H(x, u, v) \geq \gamma_{4}|u|^{\alpha}-\delta \lambda_{1} v^{2}$ for all $(x, z)$;

$\left(H_{6}\right)$ if $q \geq 2^{*}$, then $H_{v}(x, z) v \geq \gamma_{5}|v|^{q}-\gamma_{6}\left(|v|+u^{2}\right)$ for all $(x, z)$.

With these assumptions we have the following three results, for the case when $p<2$.

Theorem 1.3. Suppose that $\left(H_{0}\right)$ holds with $p<2$ and $q \geq 2$. If $H(x, z)$ also satisfies $\left(H_{4}\right)-\left(H_{6}\right)$, then $(\mathrm{E})$ has at least one nontrivial solution.

Theorem 1.4. Suppose that $H(x, z)$ is even in $z$ and $\left(H_{0}\right)$ holds with $p<2$ and $q \geq 2$. If $H(x, z)$ also satisfies $\left(H_{4}\right)-\left(H_{6}\right)$, then $(\mathrm{E})$ has a sequence $\left(z_{n}\right)$ of solutions with negative energies $I\left(z_{n}\right)$ going to 0 as $n \rightarrow \infty$.

Theorem 1.5. Let $\left(H_{0}\right)$, with $p, q \in(1,2)$, and $\left(H_{5}\right)$ be satisfied. Then $(\mathrm{E})$ has at least one nontrivial solution. If, in addition, $H(x, z)$ is even in $z$, then (E) has a sequence $\left(z_{n}\right)$ of solutions with negative energies $I\left(z_{n}\right)$ going to 0 as $n \rightarrow \infty$.

Finally, we consider the case when $p=2$, which presents some sort of resonance. Assume

$\left(H_{7}\right)$ there exist $b_{0} \leq 0<a_{0}$ such that $R_{0}(x, z):=H(x, z)-\frac{1}{2}\left(a_{0} u^{2}+b_{0} v^{2}\right)=$ $o\left(|z|^{2}\right)$ as $z \rightarrow 0$ uniformly in $x$;

$\left(H_{8}\right)$ there exist $\sigma \in(1,2), a_{\infty} \in\left[a_{0}, \infty\right) \backslash \sigma(-\Delta)$, such that $R_{\infty}(x, z):=$ $H(x, z)-\frac{1}{2} a_{\infty} u^{2}$ satisfies $\left|\partial_{u} R_{\infty}(x, z)\right| \leq \gamma_{7}\left(1+|u|^{\sigma-1}+|v|^{\tau-1}\right)$ and $R_{\infty}(x, z)$ $\geq \gamma_{8}|v|^{q}-\gamma_{9}\left(1+|u|^{\sigma}\right)$.

The position of the numbers $a_{0}, a_{\infty}, b_{0}$ with respect to the spectrum $\sigma(-\Delta)$ plays a very essential role in the next result. For that matter, let $i, j, k$ be nonnegative integers such that $\lambda_{i}=\min \left\{\lambda \in \sigma(-\Delta): \lambda>a_{0}\right\}, \lambda_{j}=\max \{\lambda \in \sigma(-\Delta): \lambda<$ $\left.-b_{0}\right\}, \lambda_{k}=\max \left\{\lambda \in \sigma(-\Delta): \lambda<a_{\infty}\right\}$, and set

$$
\ell= \begin{cases}j & \text { if } a_{\infty}=a_{0}, \\ j+k-i+1 & \text { if } a_{\infty}>a_{0} .\end{cases}
$$

Now we can state our last result.

Theorem 1.6. Let $\left(H_{0}\right)$ be satisfied with $p=2$ and $\tau<1+q / 2$. Assume that $H(x, z)$ is even in $z$ and satisfies $\left(H_{7}\right)$ and $\left(H_{8}\right)$. Then $(\mathrm{E})$ has at least one pair of nontrivial solutions if $\ell=1$, and infinitely many solutions if $\ell \geq 2$.

The cases covered in Theorem 1.6 include some asymptotically linear systems. Such systems have been studied in [5], [6] and Silva [13]. However, their results are not comparable with the ones obtained here. 
We organize the paper as follows. In order to establish multiplicity of solutions we need some new abstract propositions on critical point theory for strongly indefinite functionals, which will be provided in Section 2. These propositions are based on certain Galerkin approximations, and we emphasize that the functionals do not satisfy the usual Palais-Smale condition. In Section 3 we study systems that are superlinear in the variable $u$, and prove Theorems 1.1 and 1.2. In Section 4 we consider systems that are sublinear in the variable $u$, and prove Theorems 1.3, 1.4 and 1.5. In both Sections 3 and 4 , the variable $v$ can have subcritical growth as well as supercritical growth. Finally, in Section 5, we consider a special asymptotically linear system and prove existence of multiple solutions.

\section{Critical points For StRongly indefinite FunCtionals}

Let $E$ be a Banach space with norm $\|\cdot\|$. Suppose that $E$ has a direct sum decomposition $E=E^{1} \oplus E^{2}$ with both $E^{1}$ and $E^{2}$ being infinite dimensional. Let $P^{1}$ denote the projection from $E$ onto $E^{1}$. Assume $\left(e_{n}^{1}\right)$ (resp. $\left.\left(e_{n}^{2}\right)\right)$ is a basis for $E^{1}$ (resp. $\left.E^{2}\right)$. Set

$$
X_{n}:=\operatorname{span}\left\{e_{1}^{1}, \cdots, e_{n}^{1}\right\} \oplus E^{2}, \quad X^{m}:=E^{1} \oplus \operatorname{span}\left\{e_{1}^{2}, \cdots, e_{m}^{2}\right\},
$$

and let $\left(X^{m}\right)^{\perp}$ denote the complement of $X^{m}$ in $E$. For a functional $I \in \mathcal{C}^{1}(E, \mathbb{R})$ we set $I_{n}:=\left.I\right|_{X_{n}}$, the restriction of $I$ on $X_{n}$. Recall that a sequence $\left(z_{j}\right) \subset E$ is said to be a $(\mathrm{PS})_{c}^{*}$ sequence if $z_{j} \in X_{n_{j}}, n_{j} \rightarrow \infty, I\left(z_{j}\right) \rightarrow c$ and $I_{n_{j}}^{\prime}\left(z_{j}\right) \rightarrow 0$ as $j \rightarrow \infty$. If any $(\mathrm{PS})_{c}^{*}$ sequence has a convergent subsequence, then we say that $I$ satisfies the $(\mathrm{PS})_{c}^{*}$ condition.

Denote the upper and lower level sets, respectively, by $I_{a}=\{z \in E: I(z) \geq$ $a\}, I^{b}=\{z \in E: I(z) \leq b\}$ and $I_{a}^{b}=I_{a} \cap I^{b} \quad$ (denote similarly $\left(I_{n}\right)_{a},\left(I_{n}\right)^{b}$ and $\left.\left(I_{n}\right)_{a}^{b}\right)$. We also set $\mathcal{K}=\left\{z \in E: I^{\prime}(z)=0\right\}, \mathcal{K}_{c}=\mathcal{K} \cap I_{c}, \mathcal{K}^{c}=\mathcal{K} \cap I^{c}$ and $\mathcal{K}_{a}^{b}=\mathcal{K}_{a} \cap \mathcal{K}^{b}$.

Proposition 2.1. Let $E$ be as above and let $I \in \mathcal{C}^{1}(E, \mathbb{R})$ be even with $I(0)=0$. In addition, suppose that, for each $m \in \mathbb{N}$, the conditions below hold:

$\left(I_{1}\right)$ there is $R_{m}>0$ such that $I(z) \leq 0$ for all $z \in X^{m}$ with $\|z\| \geq R_{m}$;

$\left(I_{2}\right)$ there are $r_{m}>0$ and $a_{m} \rightarrow \infty$ such that $I(z) \geq a_{m}$ for all $z \in\left(X^{m-1}\right)^{\perp}$ with $\|z\|=r_{m}$;

$\left(I_{3}\right) I$ is bounded from above on bounded sets of $X^{m}$;

$\left(I_{4}\right)$ if $c \geq 0$, any $(\mathrm{PS})_{c}^{*}$ sequence $\left(z_{n}\right)$ has a subsequence along which $z_{n} \rightarrow z \in$ $\mathcal{K}_{c}$.

Then the functional I has a sequence $\left(c_{k}\right)$ of critical values, with the property that $c_{k} \rightarrow \infty$.

Remark 2.1. This proposition is more or less known if the condition $\left(I_{4}\right)$ is replaced by the (PS)* condition (cf. [1], 9]), or by the usual Palais-Smale condition, that is, any sequence $\left(z_{k}\right) \subset E$ such that $\left|I\left(z_{k}\right)\right| \leq c$ and $I^{\prime}\left(z_{k}\right) \rightarrow 0$ has a convergent subsequence (cf. [3]).

Proposition 2.2. Let $E$ be as above and let $I \in \mathcal{C}^{1}(E, \mathbb{R})$ be even. Assume that $I(0)=0$ and that, for each $m \in \mathbb{N}$, the two conditions below hold:

$\left(I_{5}\right)$ there are $r_{m}>0$ and $a_{m}>0$ such that $I(z) \geq a_{m}$ for all $z \in X^{m}$ with $\|z\|=r_{m}$

$\left(I_{6}\right)$ there is $b_{m}>0$ with $b_{m} \rightarrow 0$ such that $I(z) \leq b_{m}$ for all $z \in\left(X^{m-1}\right)^{\perp}$. 
Moreover, suppose that either I satisfies the $(\mathrm{PS})_{c}^{*}$ condition for all $c>0$, or that the condition below holds:

$\left(I_{7}\right) \inf I(\mathcal{K})=0$, and, for all $c \geq 0$, any $(\mathrm{PS})_{c}^{*}$ sequence $\left(z_{n}\right)$ has a subsequence along which $z_{n} \rightarrow z \in \mathcal{K}^{c}$ with $z=0$ only if $c=0$.

Then I has a sequence $\left(c_{k}\right)$ of positive critical values satisfying $c_{k} \rightarrow 0$.

Proof. Let $\Sigma$ be the family of symmetric, closed subsets of $E \backslash\{0\}$, and let $\gamma: \Sigma \rightarrow$ $\mathbb{N} \cup\{0, \infty\}$ denote the Krasnoselski genus map. Set

$$
c_{n}^{m}:=\sup _{A \in \Sigma_{n}^{m}} \inf _{z \in A} I(z)
$$

where

$$
\Sigma_{n}^{m}:=\left\{A \in \Sigma: A \subset X_{n} \text { and } \gamma(A) \geq n+m\right\} .
$$

Fix $m \in \mathbb{N}$. The Borsuk-Ulam theorem implies that $A \cap\left(X^{m-1}\right)^{\perp} \neq \emptyset$ for each $A \in \Sigma_{n}^{m}$. It follows from $\left(I_{6}\right)$ that

$$
\inf _{z \in A} I(z) \leq \sup _{z \in\left(X^{m-1}\right)^{\perp}} I(z) \leq b_{m} .
$$

On the other hand, since $\gamma\left(\partial B_{r_{m}} \cap X_{n}^{m}\right)=n+m$, one has $S_{n}^{m}:=\partial B_{r_{m}} \cap X_{n}^{m} \in \Sigma_{n}^{m}$, and so, by $\left(I_{5}\right)$, we obtain

$$
\inf _{z \in S_{n}^{m}} I(z) \geq a_{m}
$$

Therefore,

$$
a_{m} \leq c_{n}^{m} \leq b_{m} .
$$

A standard deformation argument, using a positive pseudo-gradient flow, yields the existence of a sequence $\left(z_{n}^{m}\right)_{n=1}^{\infty}$, with $z_{n}^{m} \in X_{n}$ satisfying

$$
\left|I\left(z_{n}^{m}\right)-c_{n}^{m}\right| \leq \frac{1}{n} \text { and }\left\|I_{n}^{\prime}\left(z_{n}^{m}\right)\right\| \leq \frac{1}{n} .
$$

We can assume that $I\left(z_{n}^{m}\right) \rightarrow c_{m}$ as $n \rightarrow \infty$. So, $\left(z_{n}^{m}\right)$ is a $(\mathrm{PS})_{c_{m}}^{*}$ sequence with

$$
a_{m} \leq c_{m} \leq b_{m} .
$$

Now, if we assume that $I$ satisfies the (PS) ${ }_{c}^{*}$ condition for $c>0$, then the conclusion follows. Next, suppose instead that $\left(I_{7}\right)$ holds. Then, along a subsequence, $z_{n}^{m} \rightarrow$ $z_{m}$ as $n \rightarrow \infty$ with $I^{\prime}\left(z_{m}\right)=0$ and $0<I\left(z_{m}\right) \leq c_{m}$. Finally, by (2.2),

$$
I\left(z_{m}\right) \leq b_{m} \rightarrow 0,
$$

and the proof is complete.

Proposition 2.3. Let $E$ be as above and let $I \in \mathcal{C}^{1}(E, \mathbb{R})$ be even with $I(0)=0$. Suppose, in addition, that the three conditions below hold:

$\left(I_{8}\right)$ there are $\ell \in \mathbb{N}$ and $r, a>0$ such that $I(z) \geq a$ for all $z \in X^{\ell}$ with $\|z\|=r$;

$\left(I_{9}\right)$ there is $b>0$ such that $\sup I\left(E^{2}\right) \leq b$;

$\left(I_{10}\right)$ any $(\mathrm{PS})_{c}^{*}, c>0$, sequence $\left(z_{n}\right)$ has a subsequence along which $z_{n} \rightarrow z \in$ $\mathcal{K}_{c}$ and $P^{1} z_{n} \rightarrow P^{1} z$.

Then $I$ has at least one pair of nontrivial critical points if $\ell=1$, and infinitely many critical points if $\ell>1$, with positive critical values. 
Proof. Let $\Sigma, \gamma, \Sigma_{n}^{m}$ and $c_{n}^{m}$ be as in the proof of Proposition 2.2. As before, by $\left(I_{8}\right)$ and $\left(I_{9}\right)$, we obtain

$$
a \leq c_{n}^{m} \leq b \text { for all } n \in \mathbb{N} \text { and } m=1, \cdots, \ell,
$$

and we find sequences $z_{n}^{m} \in X_{n}$ such that, going to subsequences if necessary, $I\left(z_{n}^{m}\right) \rightarrow c_{m}$ and $I_{n}^{\prime}\left(z_{n}^{m}\right) \rightarrow 0$ as $n \rightarrow \infty$ with

$$
b \geq c_{1} \geq c_{2} \geq \cdots \geq c_{\ell} \geq a .
$$

Using $\left(I_{10}\right)$, we can assume furthermore that $z_{n}^{m} \rightarrow z_{m} \in \mathcal{K}_{c_{m}}$ for $m=1, \cdots, \ell$, as $n \rightarrow \infty$. If $\ell=1$ the proof is complete.

Consider $\ell>1$. Let $F=\{z \in \mathcal{K}: I(z)>0\}$. We are going to prove that $F$ is an infinite set. Arguing by contradiction, we suppose that $F$ is finite. Choose $0<\mu<a \leq b<\nu$ satisfying

$$
\mu<\inf I(F) \leq \sup I(F)<\nu .
$$

Let $k \in \mathbb{N}$ be so large that $0 \notin A:=Q^{k} F$, where $Q^{k}: E \rightarrow X^{k}$ denotes the projection. Then $A$ is also finite, and $\gamma(A)=1$. By the continuity of $\gamma$, for all $\delta>0$ small, $\gamma\left(N_{\delta}^{k}(A)\right)=\gamma(A)$, where $N_{\delta}^{k}(A)=\left\{z \in X^{k}: \operatorname{dist}(z, A) \leq \delta\right\}$. Set $C_{\delta}=N_{\delta}^{k}(A) \oplus\left(X^{k}\right)^{\perp}$. Since $N_{\delta}^{k}(A) \subset C_{\delta}$ and $Q^{k}: C_{\delta} \rightarrow N_{\delta}^{k}(A)$, it follows from the properties of $\gamma$ that $\gamma\left(C_{\delta}\right)=\gamma\left(N_{\delta}^{k}(A)\right)$. We remark that $Q^{k}=P^{1}+\left(Q^{k}-P^{1}\right)$ and that the range of $Q^{k}-P^{1}$ is $k$-dimensional. So by virtue of $\left(I_{10}\right)$, we conclude that, for all $c \geq 0$, any $(\mathrm{PS})_{c}^{*}$ sequence $\left(z_{n}\right)$ has a subsequence along which $z_{n} \rightarrow z \in \mathcal{K}_{c}$ and $Q^{k} z_{n} \rightarrow Q^{k} z$. Hence there are $n_{0} \in \mathbb{N}$ and $\sigma>0$ such that for all $n \geq n_{0}$,

$$
\left\|I_{n}^{\prime}(w)\right\| \geq \sigma \quad \text { for all } w \in\left(I_{n}\right)_{\mu}^{\nu} \backslash C_{\delta}^{n},
$$

where $C_{\delta}^{n}=C_{\delta} \cap X_{n}$. By a standard deformation argument, we can then construct a sequence of odd homeomorphisms $\eta_{n}: X_{n} \rightarrow X_{n}$ such that

$$
\eta_{n}\left(\left(I_{n}\right)_{\mu} \backslash C_{\delta}^{n}\right) \subset\left(I_{n}\right)_{\nu}
$$

(cf. [12]). For $n_{0}$ sufficiently large, we can suppose that

$$
\mu<c_{n}^{\ell} \leq c_{n}^{\ell-1} \leq \cdots \leq c_{n}^{1}<\nu \text { for all } n \geq n_{0} .
$$

Let $G \in \Sigma_{n}^{\ell}$ be such that $\inf I(G)>\left(\mu+c_{n}^{\ell}\right) / 2$. One then has

$$
\eta_{n}\left(G \backslash C_{\delta}^{n}\right) \subset\left(I_{n}\right)_{\nu}
$$

and

$$
\begin{aligned}
\gamma\left(\eta_{n}\left(G \backslash C_{\delta}^{n}\right)\right) & =\gamma\left(G \backslash C_{\delta}^{n}\right) \geq \gamma(G)-\gamma\left(C_{\delta}^{n}\right) \\
& \geq n+\ell-\gamma\left(C_{\delta}^{n}\right) \geq n+\ell-1 .
\end{aligned}
$$

Thus $\eta_{n}\left(G \backslash C_{\delta}^{n}\right) \in \Sigma_{n}^{\ell-1}$ and $\nu \leq \inf I\left(\eta_{n}\left(G \backslash C_{\delta}^{n}\right)\right) \leq{c_{n}^{\ell-1}}^{\ell}$. One finally comes to $\nu \leq c_{n}^{\ell-1}<\nu$, which is a contradiction.

From now on we turn to the system (E). We denote by $|\cdot|_{t}$ the usual $L^{t}(\Omega)$ norm for all $t \in[1, \infty]$. For $q>1$ let $V_{q}=H_{0}^{1}(\Omega)$ if $q \leq 2^{*}$ and $V_{q}=H_{0}^{1}(\Omega) \cap L^{q}(\Omega)$, the Banach space equipped with the norm $\|v\|_{V_{q}}=\left(|\nabla v|_{2}^{2}+|v|_{q}^{2}\right)^{1 / 2}$, if $q>2^{*}$. Let $E_{q}$ be the product space $H_{0}^{1}(\Omega) \times V_{q}$ with elements denoted by $z=(u, v)$. We denote the norm in $E_{q}$ by $\|z\|_{q}=\left(|\nabla u|_{2}^{2}+\|v\|_{V_{q}}^{2}\right)^{1 / 2}$. $E_{q}$ has the direct sum decomposition

$$
E_{q}=E_{q}^{-} \oplus E^{+}, \quad z=z^{-}+z^{+}
$$

where

$$
E_{q}^{-}=\{0\} \times V_{q} \quad \text { and } \quad E^{+}=H_{0}^{1}(\Omega) \times\{0\} .
$$


For convenience, we will write $z^{+}=u$ and $z^{-}=v$. Recall that by $\left(\lambda_{n}\right)_{n \in \mathbb{N}}$ we denote the sequence of eigenvalues of $\left(-\Delta, H_{0}^{1}(\Omega)\right)$. Let $e_{n},\left|e_{n}\right|_{2}=1$, be the eigenfunction corresponding to $\lambda_{n}$ for each $n \in \mathbb{N}$. Clearly, $e_{n}^{+}:=\left(e_{n}, 0\right), n \in \mathbb{N}$, is a basis for $E^{+}$, and $e_{n}^{-}=\left(0, e_{n}\right), n \in \mathbb{N}$, is a basis for $E_{q}^{-}$.

Suppose that the assumption $\left(H_{0}\right)$ holds. Then

$$
H(x, z) \leq c\left(1+|u|^{2^{*}}+|v|^{q}\right) \text { for all }(x, z) .
$$

So the functional

$$
I(z):=\frac{1}{2} \int_{\Omega}\left(|\nabla u|^{2}-|\nabla v|^{2}\right)-\int_{\Omega} H(x, z)
$$

is well defined in $E_{q}$. Moreover, $I \in \mathcal{C}^{1}\left(E_{q}, \mathbb{R}\right)$, and the critical points of $I$ are the solutions of $(\mathrm{E})$.

Lemma 2.1. If $\left(H_{0}\right)$ holds, then $I^{\prime}$ is weakly sequentially continuous, that is, $I^{\prime}\left(z_{n}\right) \rightarrow I^{\prime}(z)$ provided $z_{n} \rightarrow z$.

Proof. If $q<2^{*}$ this statement is well known. Assume now that $q \geq 2^{*}$. Let $z_{n} \rightarrow z$ in $E_{q}$. Clearly, for all $w=(\varphi, \psi) \in E_{q}$, we have

$$
\int_{\Omega}\left(\nabla u_{n} \nabla \varphi-\nabla v_{n} \nabla \psi\right) \rightarrow \int_{\Omega}(\nabla u \nabla \varphi-\nabla v \nabla \psi) .
$$

So it remains to show that

$$
\int_{\Omega} H_{u}\left(x, z_{n}\right) \varphi \rightarrow \int_{\Omega} H_{u}(x, z) \varphi \text { for all } \varphi \in H_{0}^{1}(\Omega)
$$

and

$$
\int_{\Omega} H_{v}\left(x, z_{n}\right) \psi \rightarrow \int_{\Omega} H_{v}(x, z) \psi \quad \text { for all } \psi \in V_{q} .
$$

By the Sobolev embedding theorem and using interpolation, we obtain that $u_{n} \rightarrow u$ in $L^{t}$ for $t \in\left[1,2^{*}\right)$ and $v_{n} \rightarrow v$ in $L^{t}$ for $t \in[1, q)$. Noting that $\left|H_{u}(x, u, v)\right| \leq \gamma_{0}\left(1+|u|^{p-1}+|v|^{\tau-1}\right)$ with $2_{*}(\tau-1)<q$, (2.5) follows easily since $u_{n} \rightarrow u$ in $L^{p}, v_{n} \rightarrow v$ in $L^{2_{*}(\tau-1)}$ and $\varphi \in H_{0}^{1}(\Omega) \subset L^{2^{*}}$. Next we see that (2.6) is clearly true when $\psi \in L^{\infty}$. In general, for a $\psi \in V_{q}$ we proceed as follows. Let $\tilde{\psi}_{m} \in L^{\infty}$ with $\tilde{\psi}_{m} \rightarrow \psi$ in $L^{q}$ as $m \rightarrow \infty$. So

$$
\left|\int_{\Omega}\left(H_{v}\left(x, z_{n}\right)-H_{v}(x, z)\right) \psi\right|=\left|\int_{\Omega}\left(H_{v}\left(x, z_{n}\right)-H_{v}(x, z)\right)\left(\tilde{\psi}_{m}+\left(\psi-\tilde{\psi}_{m}\right)\right)\right|,
$$

and using $\left(H_{0}\right)$ we see that this expression is less than the following sum:

$$
\begin{aligned}
& \left|\int_{\Omega}\left(H_{v}\left(x, z_{n}\right)-H_{v}(x, z)\right) \tilde{\psi}_{m}\right| \\
+ & c_{1}\left(\left|\psi-\tilde{\psi}_{m}\right|_{1}+\left|u_{n}\right|_{p}^{p-1}\left|\tilde{\psi}_{m}-\psi\right|_{p}+\left|v_{n}\right|_{q}^{q-1}\left|\tilde{\psi}_{m}-\psi\right|_{q}\right),
\end{aligned}
$$

which by its turn is estimated by

$$
\left|\int_{\Omega}\left(H_{v}\left(x, z_{n}\right)-H_{v}(x, z)\right) \tilde{\psi}_{m}\right|+c_{2}\left(\left|\tilde{\psi}_{m}-\psi\right|_{p}+\left|\tilde{\psi}_{m}-\psi\right|_{q}\right),
$$

since $\left(z_{n}\right)$ is bounded in $E_{q}$ and $L^{\infty}$ is dense in $L^{q}$. So (2.6) is proved, and it follows that

$$
I^{\prime}\left(z_{n}\right) w \rightarrow I^{\prime}(z) w \text { for all } w \in E_{q}
$$




\section{THE CASE $p>2$}

Throughout this section let $\left(H_{0}\right)$ be satisfied with $p>2$, and assume that $\left(H_{1}\right)$ and $\left(H_{2}\right)$ hold. Observe that, by $\left(H_{2}\right)$, there exists $R>0$ such that $H(x, z)>0$ whenever $|z| \geq R$. This, jointly with $\left(H_{1}\right)$, implies

$$
H(x, z) \geq c_{1}\left(|u|^{\mu}+|v|^{\nu}\right)-c_{2} \text { for all }(x, z)
$$

(see [10]). This, together with $(2.3)$ and $\left(H_{2}\right)$, shows that

$$
\nu \leq q \text { and } \beta \leq q .
$$

Moreover, by virtue of (3.1) and $\left(H_{2}\right)$, we may assume, without loss of generality, that (since $\mu>2$ )

$$
\alpha>2 .
$$

Now we set $E^{1}=E_{q}^{-}, E^{2}=E^{+}$and $e_{n}^{1}=e_{n}^{-}, e_{n}^{2}=e_{n}^{+}$for all $n \in \mathbb{N}$. So $E_{q}=E_{1} \oplus E_{2}$. Consider the functional defined by (2.4), which has the properties stated in Section 2.

Lemma 3.1. Any $(\mathrm{PS})_{c}^{*}$ sequence is bounded.

Proof. Let $z_{n} \in X_{n}$ be such that

$$
I\left(z_{n}\right) \rightarrow c \quad \text { and } \quad I_{n}^{\prime}\left(z_{n}\right) \rightarrow 0 .
$$
have

Case 1: $q \leq 2$. In this case $E_{q}=\left(H_{0}^{1}(\Omega)\right)^{2}$. By $\left(H_{1}\right)$, for $w_{n}:=\left(\frac{1}{\mu} u_{n}, \frac{1}{\nu} v_{n}\right)$, we

$$
\begin{aligned}
& I\left(z_{n}\right)-I_{n}^{\prime}\left(z_{n}\right) w_{n} \\
= & \left(\frac{1}{2}-\frac{1}{\mu}\right)\left|\nabla u_{n}\right|_{2}^{2}+\left(\frac{1}{\nu}-\frac{1}{2}\right)\left|\nabla v_{n}\right|_{2}^{2} \\
& +\int_{\Omega}\left(\frac{1}{\mu} H_{u}\left(x, z_{n}\right) u_{n}+\frac{1}{\nu} H_{v}\left(x, z_{n}\right) v_{n}-H\left(x, z_{n}\right)\right)-c_{1} \\
\geq & \left(\frac{1}{2}-\frac{1}{\mu}\right)\left|\nabla u_{n}\right|_{2}^{2}+\left(\frac{1}{\nu}-\frac{1}{2}\right)\left|\nabla v_{n}\right|_{2}^{2}-c_{2} .
\end{aligned}
$$

If $q<2$, then (3.2) shows that $\nu<2$, and so $\left\|z_{n}\right\|_{q}^{2} \leq c_{3}\left(1+\left\|z_{n}\right\|_{q}\right)$, which implies that $\left(z_{n}\right)$ is bounded in $E_{q}$. Assume $q=2$. Invoking (3.2), we get $\nu \leq 2$, and so $\left|\nabla u_{n}\right|_{2}^{2} \leq c\left(1+\left\|z_{n}\right\|_{q}\right)$ by (3.4). Since $H(x, z)>0$ for all $|z|$ large, and

$$
\frac{1}{2}\left|\nabla v_{n}\right|_{2}^{2}+\int_{\Omega} H\left(x, z_{n}\right)=-I\left(z_{n}\right)+\frac{1}{2}\left|\nabla u_{n}\right|_{2}^{2} \leq c\left(1+\left\|z_{n}\right\|_{q}\right),
$$

one sees that $\left\|z_{n}\right\|_{q}^{2} \leq c\left(1+\left\|z_{n}\right\|_{q}\right)$. Hence, $\left(z_{n}\right)$ is bounded.

Case 2: $q>2$. Note that in this case $\nu=\mu>2$ in $\left(H_{1}\right)$. So

$$
\begin{aligned}
I\left(z_{n}\right)-\frac{1}{2} I_{n}^{\prime}\left(z_{n}\right) z_{n} & =\int_{\Omega}\left(\frac{1}{2} H_{z}\left(x, z_{n}\right) z_{n}-H\left(x, z_{n}\right)\right) \\
& \geq\left(\frac{\mu}{2}-1\right) \int_{\Omega} H\left(x, z_{n}\right)-c,
\end{aligned}
$$

which, together with $\left(H_{2}\right)$, yields

$$
\left|u_{n}\right|_{\alpha}^{\alpha}+\left|v_{n}\right|_{\beta}^{\beta} \leq c\left(1+\left\|z_{n}\right\|_{q}\right) .
$$


Using $\left(H_{0}\right)$, we get

$$
\begin{aligned}
\left|\nabla u_{n}\right|_{2}^{2} & =I_{n}^{\prime}\left(z_{n}\right)\left(u_{n}, 0\right)+\int_{\Omega} H_{u}\left(x, z_{n}\right) u_{n} \\
& \leq c_{1}\left\|z_{n}\right\|_{q}+c_{2} \int_{\Omega}\left(\left|u_{n}\right|^{p}+\left|v_{n}\right|^{\tau-1}\left|u_{n}\right|\right) .
\end{aligned}
$$

Next we estimate the integrals in the right side of (3.7). Since $2_{*}(p-1) \leq \alpha \leq p$, we have that $\theta:=\alpha /(1+\alpha-p) \leq 2^{*}$. Using the Hölder inequality, the Sobolev embedding theorem and (3.6), we obtain

$$
\int_{\Omega}\left|u_{n}\right|^{p} \leq\left|u_{n}\right|_{\alpha}^{p-1}\left|u_{n}\right|_{\theta} \leq c_{1}+c_{2}\left\|z_{n}\right\|_{q}^{1+(p-1) / \alpha}
$$

Similarly, since $\tau-1<\beta / 2_{*}$, we have $1<\omega:=\beta /(1+\beta-\tau)<2^{*}$, and hence

$$
\int_{\Omega}\left|v_{n}\right|^{\tau-1}\left|u_{n}\right| \leq\left|v_{n}\right|_{\beta}^{\tau-1}\left|u_{n}\right|_{\omega} \leq c_{1}+c_{2}\left\|z_{n}\right\|_{q}^{1+(\tau-1) / \beta} .
$$

Therefore, using the estimate in (3.7), we obtain

$$
\left|\nabla u_{n}\right|_{2}^{2} \leq c\left(1+\left\|z_{n}\right\|_{q}^{1+(p-1) / \alpha}+\left\|z_{n}\right\|_{q}^{1+(\tau-1) / \beta}\right) .
$$

Since

$$
\left|\nabla v_{n}\right|_{2}^{2}=-I_{n}^{\prime}\left(z_{n}\right)\left(0, v_{n}\right)-\int_{\Omega} H_{z}\left(x, z_{n}\right) z_{n}+\int_{\Omega} H_{u}\left(x, z_{n}\right) u_{n},
$$

and using (3.5) and the above arguments, we obtain

$$
\left|\nabla v_{n}\right|_{2}^{2} \leq c\left(1+\left\|z_{n}\right\|_{q}^{1+(p-1) / \alpha}+\left\|z_{n}\right\|_{q}^{1+(\tau-1) / \beta}\right) .
$$

Recall that, in view of our assumptions, $(p-1) / \alpha \leq 1 / 2_{*},(\tau-1) / \beta<1 / 2_{*}$, and $\beta=q$ if $q>2^{*}$. Hence, it follows from (3.6) and (3.8)-(3.9) that $\left(z_{n}\right)$ is bounded in $E_{q}$.

Lemma 3.2. Let $z_{n} \in X_{n}$ be a $(\mathrm{PS})_{c}^{*}$ sequence. If $q \leq 2^{*}$, then $\left(z_{n}\right)$ contains a convergent subsequence. If $q>2^{*}$, then there is a $z \in E_{q}$ such that, along a subsequence, $z_{n} \rightarrow z$ and $I^{\prime}(z)=0$ and $I(z) \geq c$.

Proof. By Lemma 3.1, $\left(z_{n}\right)$ is bounded. We can assume that $z_{n} \rightarrow z$ in $E_{q}, z_{n} \rightarrow z$ in $\left(L^{s}(\Omega)\right)^{2}$ for all $1 \leq s<2^{*}$, and $z_{n}(x) \rightarrow z(x)$ a.e. on $\Omega$. It follows from the weak sequential continuity of $I^{\prime}$ (see Lemma 2.1) that $I^{\prime}(z)=0$. Since $I_{n}^{\prime}\left(z_{n}\right) \rightarrow 0$, we obtain

$$
\begin{aligned}
\left(\nabla u_{n}, \nabla u_{n}-\nabla u\right)_{L^{2}} & =I_{n}^{\prime}\left(z_{n}\right)\left(u_{n}-u, 0\right)+\int_{\Omega} H_{u}\left(x, z_{n}\right)\left(u_{n}-u\right) \\
& =o(1)+\int_{\Omega} H_{u}\left(x, z_{n}\right)\left(u_{n}-u\right) .
\end{aligned}
$$

Using $\left(H_{0}\right)$ and the Hölder inequality, we obtain the estimate

$$
\begin{aligned}
& \left|\int_{\Omega} H_{u}\left(x, z_{n}\right)\left(u_{n}-u\right)\right| \\
\leq & c\left(\left|u_{n}-u\right|_{1}+\left|u_{n}\right|_{p}^{p-1}\left|u_{n}-u\right|_{p}+\left|v_{n}\right|_{\beta}^{\tau-1}\left|u_{n}-u\right|_{\omega}\right)=o(1),
\end{aligned}
$$

where $\omega$ is as in the proof of Lemma 3.1. Hence $\left|\nabla u_{n}\right|_{2}^{2} \rightarrow|\nabla u|_{2}^{2}$, which implies $u_{n} \rightarrow u$ in $H_{0}^{1}(\Omega)$. Let $P_{n}: E_{q} \rightarrow X_{n}$ denote the projection. Observe that $P_{n} z \rightarrow z$ 
in $E_{q}$ for all $z \in E_{q}$. Moreover, using again $\left(H_{0}\right)$ and the Hölder inequality, we estimate

$$
\begin{aligned}
& \left|\int_{\Omega} H_{v}\left(x, z_{n}\right)\left(v-P_{n} v\right)\right| \\
\leq & c\left(\left|v-P_{n} v\right|_{1}+\left|u_{n}\right|_{p}^{p-1}\left|v-P_{n} v\right|_{p}+\left|v_{n}\right|_{q}^{q-1}\left|v-P_{n} v\right|_{q}\right) \rightarrow 0 .
\end{aligned}
$$

On the other hand,

$$
\begin{aligned}
\left(\nabla v_{n}, \nabla v-\nabla v_{n}\right)_{L^{2}} & =o(1)+I_{n}^{\prime}\left(z_{n}\right)\left(0, v_{n}-P_{n} v\right)+\int_{\Omega} H_{v}\left(x, z_{n}\right)\left(v_{n}-P_{n} v\right) \\
& =o(1)+\int_{\Omega} H_{v}\left(x, z_{n}\right)\left(v_{n}-v\right) \\
& =o(1)+\int_{\Omega} H_{z}\left(x, z_{n}\right)\left(z_{n}-z\right)-\int_{\Omega} H_{u}\left(x, z_{n}\right)\left(u_{n}-u\right) \\
& =o(1)+\int_{\Omega} H_{z}\left(x, z_{n}\right) z_{n}-\int_{\Omega} H_{z}\left(x, z_{n}\right) z .
\end{aligned}
$$

Lebesgue's theorem and the weak sequential continuity of $H_{z}(x, \cdot)$ (see the proof of Lemma 2.1) yield

$$
|\nabla v|_{2}^{2}-\limsup _{n \rightarrow \infty}\left|\nabla v_{n}\right|_{2}^{2}=\liminf _{n \rightarrow \infty}\left(\int_{\Omega} H_{z}\left(x, z_{n}\right) z_{n}-\int_{\Omega} H_{z}\left(x, z_{n}\right) z\right) \geq 0,
$$

i.e., $|\nabla v|_{2}^{2} \geq \lim \sup _{n \rightarrow \infty}\left|\nabla v_{n}\right|_{2}^{2}$. This, together with the weak lower semicontinuity of norms, implies $\left|\nabla v_{n}\right|_{2} \rightarrow|\nabla v|_{2}$. So $v_{n} \rightarrow v$ in $H_{0}^{1}(\Omega)$.

Therefore, if $q \leq 2^{*}$, we obtain that, along a subsequence, $z_{n} \rightarrow z$ in $E_{q}$ and consequently $I(z)=c$. Next assume that $q>2^{*}$. Observe that

$$
\begin{aligned}
I(z)-I\left(z_{n}\right)= & \frac{1}{2}\left(|\nabla u|_{2}^{2}-\left|\nabla u_{n}\right|_{2}^{2}\right)-\frac{1}{2}\left(|\nabla v|_{2}^{2}-\left|\nabla v_{n}\right|_{2}^{2}\right) \\
& +\int_{\Omega} H\left(x, z_{n}\right)-\int_{\Omega} H(x, z) ;
\end{aligned}
$$

hence,

$$
I(z)-c=o(1)+\int_{\Omega} H\left(x, z_{n}\right)-\int_{\Omega} H(x, z) .
$$

Lebesgue's theorem then yields

$$
I(z)-c=\liminf _{n \rightarrow \infty} \int_{\Omega} H\left(x, z_{n}\right)-\int_{\Omega} H(x, z) \geq 0,
$$

that is, $I(z) \geq c$.

Lemma 3.3. If $\left(H_{3}\right)$ also holds, there are $r, \rho>0$ such that $\inf I\left(\partial B_{r} E^{+}\right) \geq \rho$.

Proof. By $\left(H_{0}\right)$ and $\left(H_{3}\right)$, for any $\varepsilon>0$, there is $c_{\varepsilon}>0$ such that

$$
H(x, u, 0) \leq \varepsilon|u|^{2}+c_{\varepsilon}|u|^{2^{*}} .
$$

Hence,

and the conclusion follows easily.

$$
I(u) \geq \frac{1}{2}|\nabla u|_{2}^{2}-\varepsilon|u|_{2}^{2}-c_{\varepsilon}|u|_{2^{*}}^{2^{*}},
$$

Let $e \in E^{+}$with $|\nabla e|_{2}^{2}=1$, and set

$$
Q=\left\{(s e, v): 0 \leq s \leq r_{1},\|v\|_{q} \leq r_{2}\right\} .
$$


Lemma 3.4. If $\left(H_{3}\right)$ also holds, there are $r_{1}, r_{2}>0$, with $r_{1}>r$, such that $I(z) \leq 0$ for all $z \in \partial Q$.

Proof. By $\left(H_{3}\right), I(z) \leq 0$ for all $z \in E_{q}^{-}$. By $\left(H_{2}\right)$,

$$
I((s e, v)) \leq \frac{s^{2}}{2}-\frac{1}{2}|\nabla v|_{2}^{2}-c_{1} \int_{\Omega}\left(|s e|^{\alpha}+|v|^{\beta}\right)+c_{2} .
$$

The conclusion follows since $\alpha>2$.

We are now in a position to prove Theorem 1.1.

Proof of Theorem 1.1. Lemmas 3.3 and 3.4 say that $I$ has the linking geometry. Let $Q_{n}:=Q \cap X_{n}$, and define

$$
c_{n}:=\inf _{\gamma \in \Gamma_{n}} \max I\left(\gamma\left(Q_{n}\right)\right),
$$

where $\Gamma_{n}:=\left\{\gamma \in \mathcal{C}\left(Q_{n}, X_{n}\right):\left.\gamma\right|_{\partial Q_{n}}=i d\right\}$. Then $\rho \leq c_{n} \leq \kappa:=\sup I(Q)$. A standard deformation argument shows that there is $z_{n} \in X_{n}$ such that $\left|I\left(z_{n}\right)-c_{n}\right| \leq$ $1 / n$ and $\left\|I_{n}^{\prime}\left(z_{n}\right)\right\| \leq 1 / n$. So we obtain a $(\mathrm{PS})_{c}^{*}$ sequence $\left(z_{n}\right)$ with $c \in[\rho, \kappa]$. Lemma 3.2 implies $z_{n} \rightarrow z$ with $I^{\prime}(z)=0$ and $I(z) \geq c$. The proof is complete.

We now consider the multiplicity of solutions using Proposition 2.1.

Lemma 3.5. I satisfies $\left(I_{1}\right)$.

Proof. Using $\left(H_{2}\right)$, we obtain

$$
I(z) \leq \frac{1}{2}|\nabla u|_{2}^{2}-\frac{1}{2}|\nabla v|_{2}^{2}-c_{1} \int_{\Omega}\left(|u|^{\alpha}+|v|^{\beta}\right)+c_{2} .
$$

Since all norms in $\operatorname{span}\left\{e_{1}, \cdots, e_{m}\right\}$ are equivalent, we obtain

$$
I(z) \leq-\left(c_{3}|\nabla u|_{2}^{\alpha-2}-\frac{1}{2}\right)|\nabla u|_{2}^{2}-\left(\frac{1}{2}|\nabla v|_{2}^{2}+c_{1}|v|_{\beta}^{\beta}\right)+c_{2}
$$

for all $z=(u, v) \in X^{m} \simeq \operatorname{span}\left\{e_{1}, \cdots, e_{m}\right\} \times V_{q}$. So $\left(I_{1}\right)$ follows easily.

Lemma 3.6. I satisfies $\left(I_{2}\right)$.

Proof. Since $\left(X^{m}\right)^{\perp} \subset H_{0}^{1}(\Omega)$ and $H_{0}^{1}(\Omega)$ embeds compactly in $L^{p}(\Omega)$, we have that $\eta_{m}>0$ and $\eta_{m} \rightarrow 0$ as $m \rightarrow \infty$, where

$$
\eta_{m}:=\sup _{u \in\left(X^{m}\right)^{\perp} \backslash\{0\}} \frac{|u|_{p}}{|\nabla u|_{2}}
$$

see Lemma 3.8 in [14]. For $z=(u, 0) \in\left(X^{m}\right)^{\perp}$, it follows from $\left(H_{0}\right)$ that

$$
\begin{aligned}
I(z) & =\frac{1}{2}|\nabla u|_{2}^{2}-\int_{\Omega} H(x, u, 0) \geq \frac{1}{2}|\nabla u|_{2}^{2}-c_{1}|u|_{p}^{p}-c_{2} \\
& \geq \frac{1}{2}|\nabla u|_{2}^{2}-c_{1} \eta_{m}^{p}|\nabla u|_{2}^{p}-c_{2} .
\end{aligned}
$$

Setting $r_{m}=\left(p c_{1} \eta_{m}^{p}\right)^{1 /(2-p)}$ and $a_{m}=(p-2) r_{m}^{2} / 2 p-c_{2}$, we come to the desired conclusion.

Proof of Theorem 1.2. Since $H(x, z)$ is even in $z, I$ is even. Lemma 3.2 shows that $I$ satisfies the assumption $\left(I_{4}\right)$ of Proposition 2.1. Lemmas 3.5 and 3.6 show that $\left(I_{1}\right)$ and $\left(I_{2}\right)$ hold. Clearly $\left(I_{3}\right)$ is also true. Therefore by Proposition 2.1, there is a sequence $\left(z_{n}\right) \subset E_{q}$ satisfying $I^{\prime}\left(z_{n}\right)=0$ and $I\left(z_{n}\right) \rightarrow \infty$. The proof is complete. 


\section{The CASE $p<2$}

Throughout this section we assume that $\left(H_{0}\right)$ is satisfied with $p \in(1,2)$. We also suppose that $\left(H_{4}\right)-\left(H_{6}\right)$ hold.

Let $E_{q}=E^{1} \oplus E^{2}$ be as in Section 3. Consider the functional

$$
J(z)=-I(z)=\int_{\Omega} H(x, z)+\frac{1}{2}|\nabla v|_{2}^{2}-\frac{1}{2}|\nabla u|_{2}^{2} .
$$

Lemma 4.1. Any $(\mathrm{PS})_{c}^{*}$ sequence $\left(z_{n}\right)$ has a subsequence converging weakly to a critical point $z$ of $J$ with $J(z) \leq c$, and $z=0$ only if $z_{n} \rightarrow 0$ in $E_{q}$.

Proof. The proof is divided into two parts.

Part $I$. The sequence $\left(z_{n}\right)$ is bounded in $E_{q}$. By $\left(H_{4}\right)$ it follows that

$$
J\left(z_{n}\right)-J_{n}^{\prime}\left(z_{n}\right)\left(\frac{1}{\mu} u_{n}, \frac{1}{\nu} v_{n}\right) \geq\left(\frac{1}{2}-\frac{1}{\nu}\right)\left|\nabla v_{n}\right|_{2}^{2}+\left(\frac{1}{\mu}-\frac{1}{2}\right)\left|\nabla u_{n}\right|_{2}^{2}-c .
$$

Hence $\left|\nabla u_{n}\right|_{2}^{2} \leq c\left(1+\left\|z_{n}\right\|_{q}\right)$. If $\nu>2$, we also get $\left|\nabla v_{n}\right|_{2}^{2} \leq c\left(1+\left\|z_{n}\right\|_{q}\right)$. If $\nu=2$, we use $\left(H_{5}\right)$ and the fact that $|\nabla v|_{2}^{2} \geq \lambda_{1}|v|_{2}^{2}$ in order to obtain

$$
\left(\frac{1}{2}-\delta\right)\left|\nabla v_{n}\right|_{2}^{2} \leq \frac{1}{2}\left|\nabla v_{n}\right|_{2}^{2}+\int_{\Omega} H\left(x, z_{n}\right)=J\left(z_{n}\right)+\frac{1}{2}\left|\nabla u_{n}\right|_{2}^{2} .
$$

Hence, $\left|\nabla v_{n}\right|_{2}^{2} \leq c\left(1+\left\|z_{n}\right\|_{q}\right)$, and we get

$$
\left|\nabla u_{n}\right|_{2}^{2}+\left|\nabla v_{n}\right|_{2}^{2} \leq c\left(1+\left\|z_{n}\right\|_{q}\right) .
$$

Thus, if $q \leq 2^{*}$, then $\left(z_{n}\right)$ is bounded in $E_{q}$. Assume next that $q>2^{*}$. It follows from $\left(H_{6}\right)$ that

$$
J_{n}^{\prime}\left(z_{n}\right)\left(0, v_{n}\right) \geq c_{1}\left|v_{n}\right|_{q}^{q}+\left|\nabla v_{n}\right|_{2}^{2}-c_{2}\left(\left|v_{n}\right|_{1}+\left|u_{n}\right|_{2}^{2}\right) .
$$

Thus $\left|\nabla u_{n}\right|_{2}^{2}+\left|\nabla v_{n}\right|_{2}^{2}+\left|v_{n}\right|_{q}^{q} \leq c\left(1+\left\|z_{n}\right\|_{q}\right)$, which implies that $\left(z_{n}\right)$ is bounded in $E_{q}$ also in the case when $q>2^{*}$.

Part II. We can now suppose that $z_{n} \rightarrow z$ in $E_{q}, z_{n} \rightarrow z$ in $\left(L^{s}(\Omega)\right)^{2}$ for all $1 \leq s<2^{*}$, and $z_{n}(x) \rightarrow z(x)$ a.e. in $x \in \Omega$. It follows that $z$ is a critical point of $J$. As in the proof of Lemma 3.2, using $\left(H_{0}\right)$ and

$$
J_{n}^{\prime}\left(z_{n}\right)\left(u_{n}-u, 0\right)=\int_{\Omega} H_{u}\left(x, z_{n}\right)\left(u_{n}-u\right)-\left(\nabla u_{n}, \nabla\left(u_{n}-u\right)\right)_{L^{2}},
$$

we obtain that

$$
\begin{aligned}
& \left|\left(\nabla u_{n}, \nabla\left(u_{n}-u\right)\right)_{L^{2}}\right| \\
\leq & o(1)+c\left(\left|u_{n}-u\right|_{1}+\left|u_{n}\right|_{p}^{p-1}\left|u_{n}-u\right|_{p}+\left|v_{n}\right|_{\beta}^{\tau-1}\left|u_{n}-u\right|_{\omega}\right)=o(1),
\end{aligned}
$$

and so $u_{n} \rightarrow u$ in $H_{0}^{1}(\Omega)$. Let $P_{n}: E_{q} \rightarrow X_{n}$ be the projection as in the proof of Lemma 3.2. So we obtain

$$
\begin{aligned}
\left(\nabla v_{n}, \nabla\left(v-v_{n}\right)\right)_{L^{2}} & =o(1)+\left(\nabla v_{n}, \nabla\left(P_{n} v-v_{n}\right)\right)_{L^{2}} \\
& =o(1)+\int_{\Omega} H_{v}\left(x, z_{n}\right)\left(v_{n}-P_{n} v\right)-J_{n}^{\prime}\left(z_{n}\right)\left(0, v_{n}-P_{n} v\right) \\
& =o(1)+\int_{\Omega} H_{v}\left(x, z_{n}\right)\left(v_{n}-v\right)+\int_{\Omega} H_{v}\left(x, z_{n}\right)\left(v-P_{n} v\right) .
\end{aligned}
$$

Using $\left(H_{0}\right)$, we have

$$
\left|\int_{\Omega} H_{v}\left(x, z_{n}\right)\left(v-P_{n} v\right)\right| \leq c\left(1+\left|u_{n}\right|_{2^{*}}^{2^{*}}+\left|v_{n}\right|_{q}^{q-1}\right)\left\|v-P_{n} v\right\|_{q} \rightarrow 0 .
$$


Consequently,

$$
\left(\nabla v_{n}, \nabla\left(v-v_{n}\right)\right)_{L^{2}}=\int_{\Omega} H_{v}\left(x, z_{n}\right)\left(v_{n}-v\right)+o(1) .
$$

Thus if $q<2^{*}$, it follows from (4.2) that $\left|\nabla v_{n}\right|_{2} \rightarrow|\nabla v|_{2}$, which implies $v_{n} \rightarrow v$, and so $z_{n} \rightarrow z$. This proves that $J$ satisfies the (PS) ${ }_{c}^{*}$ condition in this case, and that $J(z)=c$.

Consider next $q \geq 2^{*}$. The weak sequential continuity of $H_{v}(x, \cdot)$ (see the proof of Lemma 2.1) yields $\int_{\Omega} H_{v}\left(x, z_{n}\right) v \rightarrow \int_{\Omega} H_{v}(x, z) v$. By $\left(H_{6}\right), f_{n}(x):=H_{v}\left(x, z_{n}\right) v_{n}+$ $\gamma_{6}\left(\left|v_{n}\right|+\left|u_{n}\right|^{2}\right) \geq 0$. Using the fact that $\left|v_{n}\right|_{1} \rightarrow|v|_{1}$ and $\left|u_{n}\right|_{2} \rightarrow|u|_{2}$, and applying Fatou's lemma to the sequence $\left(f_{n}\right)$, we get

$$
\liminf _{n \rightarrow \infty} \int_{\Omega} H_{v}\left(x, z_{n}\right) v_{n} \geq \int_{\Omega} H_{v}(x, z) v .
$$

Using this estimate in (4.2), we obtain that $|\nabla v|_{2}^{2} \geq \lim \sup _{n \rightarrow \infty}\left|\nabla v_{n}\right|_{2}^{2}$, which implies that $v_{n} \rightarrow v$ in $H_{0}^{1}(\Omega)$. In order to conclude that $J(z) \leq c$, we use the estimate

$$
J\left(z_{n}\right)-J(z)=\int_{\Omega}\left(H\left(x, z_{n}\right)-H(x, z)\right)+o(1),
$$

$\left(H_{4}\right)$ and Fatou's lemma. Finally, if $z=0$, then $z_{n} \rightarrow 0$ in $\left(H_{0}^{1}(\Omega)\right)^{2}$. By (4.1),

$$
\left|v_{n}\right|_{q}^{q} \leq o(1)+c\left(\left|v_{n}\right|_{1}+\left|u_{n}\right|_{2}^{2}\right) \rightarrow 0
$$

and so $z_{n} \rightarrow 0$.

Remark 4.1. In a similar way, using even simpler arguments, one checks that, if $\left(H_{0}\right)$ holds with $p, q \in(1,2), J$ satisfies the $(\mathrm{PS})_{c}^{*}$ condition for all $c$.

Remark 4.2. Let $\tilde{J}_{m}=\left.J\right|_{X^{m}}$ denote the restriction of $J$ on $X^{m}$. As in Lemma 4.1, it is not difficult to check that, if the sequence $\left(z_{m}\right) \subset E_{q}$, with $z_{m} \in X^{m}$, satisfies $J\left(z_{m}\right) \rightarrow c$ and $\tilde{J}_{m}^{\prime}\left(z_{m}\right) \rightarrow 0$ as $m \rightarrow \infty$, then it possesses a subsequence converging weakly to a critical point $z$ of $J$ with $J(z) \leq c$, and $z=0$ only if $z_{n} \rightarrow 0$ in $E_{q}$. We also have, as in Remark 4.1, that, if $\left(H_{0}\right)$ holds with $p, q \in(1,2)$, then any such sequence has a convergent subsequence.

Lemma 4.2. There is an $R>0$ such that $J(z) \leq 0$ for all $z=(u, 0)$ with $\|z\| \geq R$.

Proof. By $\left(H_{0}\right)$, we have $H(x, u, 0) \leq c\left(1+|u|^{p}\right)$. Hence

$$
\begin{aligned}
J((u, 0)) & =\int_{\Omega} H(x, u, 0)-\frac{1}{2}|\nabla u|_{2}^{2} \leq c_{1}+c_{2}|u|_{p}^{p}-\frac{1}{2}|\nabla u|_{2}^{2} \\
& \leq c_{1}-\left(\frac{1}{2}|\nabla u|_{2}^{2-p}-c_{3}\right)|\nabla u|_{2}^{p},
\end{aligned}
$$

and the lemma follows, since $p<2$.

Lemma 4.3. For $\varepsilon>0$ small there is $\rho>0$ such that $J\left(\left(\varepsilon e_{1}, v\right)\right) \geq \rho$ for all $v \in V_{q}$, where $e_{1}$ is the eigenfunction corresponding to the first eigenvalue $\lambda_{1}$ of $\left(-\Delta, H_{0}^{1}(\Omega)\right)$.

Proof. By $\left(H_{5}\right)$, for $\varepsilon>0$ small, $H\left(x, \varepsilon e_{1}, v\right) \geq \gamma_{4} \varepsilon^{\alpha} e_{1}^{\alpha}-\delta \lambda_{1} v^{2}$; hence,

$$
J\left(\left(\varepsilon e_{1}, v\right)\right)=\int_{\Omega} H\left(x, \varepsilon e_{1}, v\right)+\frac{1}{2}|\nabla v|_{2}^{2}-\frac{1}{2} \lambda_{1} \varepsilon^{2} \geq\left(\gamma_{4}\left|e_{1}\right|_{\alpha}^{\alpha}-\frac{1}{2} \lambda_{1} \varepsilon^{2-\alpha}\right) \varepsilon^{\alpha} .
$$

The conclusion follows. 
We are now ready to prove Theorem 1.3.

Proof of Theorem 1.3. Recall that $X^{m} \simeq \operatorname{span}\left\{e_{1}, \cdots, e_{m}\right\} \times V_{q}$, and consider the restrictions $\tilde{J}_{m}$ as defined in Remark 4.2. Set $D_{R}=B_{R} \cap E^{2}=B_{R} \cap\left(H_{0}^{1}(\Omega) \times\{0\}\right)$ and $D_{m}=D_{R} \cap X^{m}$, where $R>0$ comes from Lemma 4.2. Define

$$
c_{m}:=\inf _{\gamma \in \Gamma_{m}} \max J\left(\gamma\left(D_{m}\right)\right),
$$

where $\Gamma_{m}:=\left\{\gamma \in \mathcal{C}\left(D_{m}, X^{m}\right): \gamma(z)=z\right.$ for all $\left.z \in \partial D_{m}\right\}$. It is well known that $\gamma\left(D_{m}\right) \cap W \neq \emptyset$ for all $\gamma \in \Gamma_{m}$, where $W=\left\{\left(\varepsilon e_{1}, 0\right)\right\} \times V_{q}$ with $\varepsilon>0$ small. Invoking Lemma 4.3, we fix an $\varepsilon>0$ so small that there is $\rho>0$ satisfying $\inf J(W) \geq \rho$. Then we have

$$
\rho \leq c_{m} \leq b:=\max J\left(D_{R}\right)
$$

The well-known saddle point theorem (cf. [12] or 4, 14]) implies that there is $z_{m} \in X^{m}$ satisfying $\left|J\left(z_{m}\right)-c_{m}\right| \leq 1 / m$ and $\left\|\tilde{J}_{m}^{\prime}\left(z_{m}\right)\right\| \leq 1 / m$. Now by virtue of Remark 4.2, along a subsequence, $z_{m} \rightarrow z$ with $J^{\prime}(z)=0$ and $z \neq 0$, ending the proof.

We now turn to the proof of Theorems 1.4 and 1.5.

Lemma 4.4. If, in addition, $\gamma_{3}=0$ in $\left(H_{4}\right)$, then $J$ satisfies $\left(I_{5}\right)$.

Proof. It follows from $\left(H_{5}\right)$ that

$$
\begin{aligned}
J(z) & \geq c_{1}|u|_{\alpha}^{\alpha}+\left(\frac{1}{2}-\delta\right)|\nabla v|_{2}^{2}-\frac{1}{2}|\nabla u|_{2}^{2} \\
& \geq\left(c_{2}-\frac{1}{2}|\nabla u|^{2-\alpha}\right)|\nabla u|_{2}^{\alpha}+\left(\frac{1}{2}-\delta\right)|\nabla v|_{2}^{2} .
\end{aligned}
$$

Since $\alpha<2$, the result follows in the case when $q \leq 2^{*}$. Next consider $q>2^{*}$. Suppose $\left(I_{5}\right)$ does not hold. Then for any $r>0$ there is a sequence $z_{j} \in X^{m}$ such that $\left\|z_{j}\right\|=r$ and $J\left(z_{j}\right) \rightarrow 0$. It follows from (4.3) with $z=z_{j}$, and for $r$ small, that $\left|\nabla u_{j}\right|_{2} \rightarrow 0$ and $\left|\nabla v_{j}\right|_{2} \rightarrow 0$. All this implies that $\int_{\Omega} H\left(x, z_{j}\right) \rightarrow 0$. From assumption $\left(H_{0}\right)$ and the fact that $\left(u_{j}\right)$ lies in a finite-dimensional subspace, it follows that $\int_{\Omega} H_{u}\left(x, z_{j}\right) u_{j} \rightarrow 0$. Consequently, by $\left(H_{4}\right)$ with $\gamma_{3}=0, \int_{\Omega} H_{v}\left(x, z_{j}\right) v_{j} \rightarrow 0$. This, jointly with $\left(H_{6}\right)$, yields

$$
\left|v_{j}\right|_{q}^{q} \leq c_{1} \int_{\Omega} H_{v}\left(x, z_{j}\right) v_{j}+c_{2}\left(\left|v_{j}\right|_{1}+\left|u_{j}\right|_{2}^{2}\right) \rightarrow 0 .
$$

Hence, $z_{j} \rightarrow 0$ in $E_{q}$, which is a contradiction.

Lemma 4.5. $J$ satisfies $\left(I_{6}\right)$.

Proof. By $\left(H_{0}\right), H(x, u, 0) \leq c\left(|u|+|u|^{p}\right)$, and so, for $u \in\left(X^{m-1}\right)^{\perp}$, one has

$$
\begin{aligned}
J((u, 0)) & \leq c_{1}\left(|u|_{p}+|u|_{p}^{p}\right)-\frac{1}{2}|\nabla u|_{2}^{2} \\
& \leq\left(c_{1}|u|_{p}-\frac{1}{4}|\nabla u|_{2}^{2}\right)+\left(c_{1}|u|_{p}^{p}-\frac{1}{4}|\nabla u|_{2}^{2}\right) \\
& \leq\left(c_{1} \eta_{m}-\frac{1}{4}|\nabla u|_{2}\right)|\nabla u|_{2}+\left(c_{1} \eta_{m}^{p}-\frac{1}{4}|\nabla u|_{2}^{2-p}\right)|\nabla u|_{2}^{p},
\end{aligned}
$$

where $\eta_{m}$ was defined by (3.10). Let $b_{m}:=\left(c_{1} \eta_{m}\right)^{2}+(1-p / 2) c_{1} \eta_{m}^{p}\left(2 p c_{1} \eta_{m}^{p}\right)^{p /(2-p)}$. Then $0<b_{m} \rightarrow 0$ and $J((u, 0)) \leq b_{m}$ for all $(u, 0) \in\left(X^{m-1}\right)^{\perp}$. 
Proof of Theorem 1.4. Since $H(x, z)$ is even in $z, J$ is even. If $q \leq 2^{*}$, then $J$ satisfies the (PS)* condition for all $c$ (see the proof of Lemma 4.1). If $q>2^{*}$, then, using assumption $\left(H_{4}\right)$ applied to a critical point $z$, we obtain

$$
J(z)=J(z)-J_{n}^{\prime}(z)\left(\frac{1}{\mu} u, \frac{1}{\nu} v\right) \geq\left(\frac{1}{2}-\frac{1}{\nu}\right)|\nabla v|_{2}^{2}+\left(\frac{1}{\mu}-\frac{1}{2}\right)|\nabla u|_{2}^{2} \geq 0 .
$$

This, jointly with Lemma 4.1, shows that $\left(I_{7}\right)$ is satisfied. It follows from Lemmas 4.4 and 4.5 that $J$ satisfies $\left(I_{5}\right)$ and $\left(I_{6}\right)$. Therefore, the desired conclusion follows.

Finally, we prove Theorem 1.5.

Proof of Theorem 1.5. The proof of the existence of one nontrivial solution is similar to that of Theorem 1.3, using Remark 4.2 and Lemmas 4.2 and 4.3. The other conclusion can be obtained along the lines of the proof of Theorem 1.4, using Remark 4.1 and Lemmas 4.4 and 4.5 .

\section{THE CASE $p=2$}

In this section we always assume that $\left(H_{0}\right)$ holds with $p=2$ and $\tau<1+q / 2$. We also suppose that $\left(H_{7}\right)$ and $\left(H_{8}\right)$ are satisfied. We will apply Proposition 2.3 in order to prove Theorem 1.6. Thus, set

$$
E^{2}=\operatorname{span}\left\{e_{1}^{+}, \cdots, e_{k}^{+}\right\} \oplus E_{q}^{-} \simeq \operatorname{span}\left\{e_{1}, \cdots, e_{k}\right\} \times V_{q}, \quad E^{1}=E_{q} \ominus E^{2},
$$

and

$$
X^{\ell}=E^{1} \oplus \operatorname{span}\left\{e_{i}^{+}, \cdots, e_{k}^{+}, e_{1}^{-}, \cdots, e_{j}^{-}\right\}
$$

One may arrange the bases as $e_{n}^{1}=e_{k+n}^{+}$for $n \in \mathbb{N}$, and $e_{n}^{2}=e_{n+i-1}^{+}$for $1 \leq n \leq$ $\ell-j, e_{n}^{2}=e_{n-\ell+j}^{-}$for $\ell-j<n \leq \ell, e_{n}^{2}=e_{n-\ell}^{+}$for $\ell<n \leq \ell+i-1$, and $e_{n}^{2}=e_{n-k}^{-}$ for $n>\ell+i-1$. Consider the functional $I$ given by (2.4).

Lemma 5.1. I satisfies $\left(I_{8}\right)$; that is, there exist $r, a>0$ such that $I(z) \geq a$ for all $z \in X^{\ell}$ with $\|z\|_{q}=r$.

Proof. Let $z=(u, v) \in X^{\ell}$. Since $v \in \operatorname{span}\left\{e_{1}, \cdots, e_{j}\right\}$, we have $v \in L^{\infty}$. By $\left(H_{0}\right)$ and $\left(H_{7}\right)$, for any $\varepsilon>0$, there exists $c_{\varepsilon}>0$ such that

$$
R_{0}(x, z) \leq \varepsilon|z|^{2}+c_{\varepsilon}\left(|u|^{2^{*}}+|v|^{q}\right) .
$$

Thus

$$
\begin{aligned}
I(z) & =\frac{1}{2}\left(|\nabla u|_{2}^{2}-a_{0}|u|_{2}^{2}\right)-\frac{1}{2}\left(|\nabla v|_{2}^{2}-b_{0}|v|_{2}^{2}\right)-\int_{\Omega} R_{0}(x, z) \\
& \geq \frac{1}{2}\left(1-\frac{a_{0}}{\lambda_{i}}\right)|\nabla u|_{2}^{2}+\frac{1}{2}\left(\frac{-b_{0}}{\lambda_{j}}-1\right)|\nabla v|_{2}^{2}-\varepsilon|z|_{2}^{2}-c_{\varepsilon}\left(|u|_{2^{*}}^{2^{*}}+|v|_{q}^{q}\right) .
\end{aligned}
$$

Now the conclusion follows easily.

Lemma 5.2. I satisfies $\left(I_{9}\right)$; that is, $\sup I\left(E^{2}\right)<\infty$. 
Proof. For $z \in E^{2}$ we have, using $\left(H_{8}\right)$, that

$$
\begin{aligned}
I(z) & =\frac{1}{2}\left(|\nabla u|_{2}^{2}-a_{\infty}|u|_{2}^{2}\right)-\frac{1}{2}|\nabla v|_{2}^{2}-\int_{\Omega} R_{\infty}(x, z) \\
& \leq-\frac{1}{2}\left(\frac{a_{\infty}}{\lambda_{k}}-1\right)|\nabla u|_{2}^{2}-\frac{1}{2}|\nabla v|_{2}^{2}+\gamma_{9}|u|_{\sigma}^{\sigma}-\gamma_{8}|v|_{q}^{q}+\gamma_{9}|\Omega| \\
& \leq-\left(\frac{1}{2}\left(\frac{a_{\infty}}{\lambda_{k}}-1\right)|\nabla u|^{2-\sigma}-c_{1}\right)|\nabla u|_{2}^{\sigma}-\left(\frac{1}{2}|\nabla v|_{2}^{2}+\gamma_{8}|v|_{q}^{q}\right)+c_{2},
\end{aligned}
$$

which implies that $I(z) \leq 0$ for all $z \in E^{2}$ with $\|z\|_{q}$ large.

Lemma 5.3. Let $c>0$. Then any $(\mathrm{PS})_{c}$ sequence is bounded.

Proof. We decompose $H_{0}^{1}(\Omega)$ as

$$
H_{0}^{1}(\Omega)=U^{-} \oplus U^{+}, \quad u=u^{-}+u^{+},
$$

where $U^{-}=\operatorname{span}\left\{e_{1}, \cdots, e_{k}\right\}$ and $U^{+}$is the orthogonal complement of $U^{-}$in $H_{0}^{1}(\Omega)$.

Let $\left(z_{n}\right)$ be a $(\mathrm{PS})_{c}^{*}$ sequence. Using the expression of $I_{n}^{\prime}$ :

$$
I_{n}^{\prime}\left(z_{n}\right) u_{n}^{+}=\left|\nabla u_{n}^{+}\right|_{2}^{2}-a_{\infty}\left|u_{n}^{+}\right|_{2}^{2}-\int_{\Omega} \partial_{u} R_{\infty}\left(x, z_{n}\right) u_{n}^{+},
$$

plus $\left(H_{8}\right)$ and the Hölder inequality, we obtain

$$
\left(1-\frac{a_{\infty}}{\lambda_{k+1}}\right)\left|\nabla u_{n}^{+}\right|_{2}^{2} \leq c_{1}\left|\nabla u_{n}^{+}\right|_{2}+\gamma_{7}\left(\left|u_{n}^{+}\right|_{1}+\left|u_{n}\right|_{\sigma}^{\sigma-1}\left|u_{n}^{+}\right|_{\sigma}+\left|v_{n}\right|_{q}^{\tau-1}\left|u_{n}^{+}\right|_{r}\right)
$$

where $r=q /(1+q-\tau)$. By assumption, $1<r<2$. It then follows from the Sobolev embedding theorems that

$$
\left(1-\frac{a_{\infty}}{\lambda_{k+1}}\right)\left|\nabla u_{n}^{+}\right|_{2}^{2} \leq c_{2}\left(1+\left|u_{n}\right|_{\sigma}^{\sigma-1}+\left|v_{n}\right|_{q}^{\tau-1}\right)\left|\nabla u_{n}^{+}\right|_{2}
$$

Similarly, we deduce that

$$
\left(\frac{a_{\infty}}{\lambda_{k}}-1\right)\left|\nabla u_{n}^{-}\right|_{2}^{2} \leq c_{2}\left(1+\left|u_{n}\right|_{\sigma}^{\sigma-1}+\left|v_{n}\right|_{q}^{\tau-1}\right)\left|\nabla u_{n}^{-}\right|_{2} .
$$

The two previous inequalities imply the estimate

$$
\left|\nabla u_{n}\right|_{2}^{2} \leq c_{3}\left(1+\left|u_{n}\right|_{\sigma}^{2(\sigma-1)}+\left|v_{n}\right|_{q}^{2(\tau-1)}\right) .
$$

Using the expression of $H$ given in $\left(H_{8}\right)$, and recalling that $I\left(z_{n}\right)>0$ for large $n$, we obtain

$$
\frac{1}{2}\left|\nabla v_{n}\right|_{2}^{2}+\int_{\Omega} R_{\infty}\left(x, z_{n}\right)=\frac{1}{2}\left|\nabla u_{n}\right|_{2}^{2}-\frac{a_{\infty}}{2}\left|u_{n}\right|_{2}^{2}-I\left(z_{n}\right) \leq \frac{1}{2}\left|\nabla u_{n}\right|_{2}^{2} .
$$

Next using (5.2), assumption $\left(H_{8}\right)$ and (5.1), we obtain

$$
\left|\nabla v_{n}\right|_{2}^{2}+c_{4}\left|v_{n}\right|_{q}^{q} \leq c_{5}\left(1+\left|u_{n}\right|_{\sigma}^{\sigma}+\left|u_{n}\right|_{\sigma}^{2(\sigma-1)}+\left|v_{n}\right|_{q}^{2(\tau-1)}\right) .
$$

The combination of (5.1) and (5.3) implies

$$
\left|\nabla z_{n}\right|_{2}^{2}+\left|v_{n}\right|_{q}^{q} \leq c_{6}\left(1+\left|u_{n}\right|_{2}^{\sigma}+\left|v_{n}\right|_{q}^{2(\tau-1)}\right) .
$$

Since $\sigma<2$ and $2(\tau-1)<q$, we see that $\left(z_{n}\right)$ is bounded.

Lemma 5.4. I satisfies $\left(I_{10}\right)$. 
Proof. Let $\left(z_{n}\right)$ be a $(\mathrm{PS})_{c}^{*}$ sequence with $c>0$. Using Lemma 5.3, an argument similar to that of Lemma 3.2 shows that along a subsequence $z_{n} \rightarrow z \in \mathcal{K}_{c}$, we have $u_{n} \rightarrow u$ in $H_{0}^{1}(\Omega)$. Since $E^{1} \subset H_{0}^{1}(\Omega)$, we have $P^{1} z_{n} \rightarrow P^{1} z$.

Proof of Theorem 1.6. Since $H(x, z)$ is even in $z, I$ is even. By assumption, $I(0)=$ 0 . Lemmas 5.1, 5.2 and 5.4 show that $I$ satisfies $\left(I_{8}\right)-\left(I_{10}\right)$. Now Proposition 2.3 applies, and the proof is complete.

\section{ACKNowledgments}

De Figueiredo was supported by CNPq-FAPESP-PRONEX. Ding was supported by the Special Funds for Major State Basic Research Projects of China, the funds of CAS/China R9902, 1001800, and the CNPq of Brazil.

\section{REFERENCES}

1. T. Bartsch, Infinitely many solutions of a symmetric Dirichlet problem, Nonlinear Anal. TMA, 20 (1993), 1205-1216. MR 94g:35093

2. T. Bartsch and D. G. De Figueiredo, Infinitely many solutions of nonlinear elliptic systems, Progress in Nonlinear Differential Equations and Their Applications, vol. 35, Birkhäuser, Basel/Switzerland, 1999, pp. 51-67. MR 2000j:35072

3. V. Benci and P. Rabinowitz, Critical point theorems for indefinite functionals, Invent. Math. 52 (1979), 241-273. MR 80i:58019

4. K. C. Chang, Infinite-Dimensional Morse Theory and Multiple Solution Problems, Birkhäuser, Boston, 1993. MR 94e:58023

5. D. G. Costa and C. A. Magalhães, A variational approach to noncooperative elliptic systems, Nonlinear Anal. TMA 25 (1995), 699-715. MR 96g:35070

6. D. G. Costa and C. A. Magalhães, A unified approach to a class of strongly indefinite functionals, J. Differential Equations 125 (1996), 521-547. MR 96m:58061

7. D. G. De Figueiredo and C. A. Magalhães, On nonquadratic Hamiltonian elliptic systems, Adv. Differential Equations 1 (1996), 881-898. MR 97f:35049

8. D. G. De Figueiredo and P. L. Felmer, On superquadratic elliptic systems, Trans. Amer. Math. Soc. 343 (1994), 97-116. MR 94g:35072

9. Y. H. Ding, Infinitely many entire solutions of an elliptic system with symmetry, Topological Methods in Nonlinear Anal. 9 (1997), 313-323. MR 99a:35062

10. P. L. Felmer, Periodic solutions of "superquadratic" Hamiltonian systems, J. Differential Equations 102 (1993), 188-207. MR 94c:58160

11. J. Hulshof and R. van der Vorst, Differential systems with strongly indefinite variational structure, J. Funct. Anal. 114 (1993), 32-58. MR 94g:35073

12. P. H. Rabinowitz, Minimax Methods in Critical Point Theory with Applications to Differential Equations, C. B. M. S. vol. 65, Amer. Math. Soc., Providence, RI, 1986. MR 87j:58024

13. E. A. B. Silva, Nontrivial solutions for noncooperative elliptic systems at resonance, Electronic J. Differential Equations 6 (2001), 267-283. MR 2001j:35097

14. M. Willem, Minimax Theorems, Progress in Nonlinear Differential Equations and their Applications, vol. 24, Birkhäuser, Boston, 1996. MR 97h:58037

IMECC-UNICAMP, Caixa Postal 6065, 13083-970 Campinas S.P. BraziL

E-mail address: djairo@ime.unicamp.br

Institute of Mathematics, AMSS, Chinese Academy of Sciences, 100080 Beijing, PeoPLE'S REPUBlic OF CHINA

E-mail address: dingyh@math03.math.ac.cn 